\title{
Intervene or Innovate: a Dilemma for Psychiatrists-in-Training
}

\author{
Nathaniel P. Morris ${ }^{1} \cdot$ Neir $^{\text {Eshel }}{ }^{1}$ \\ Received: 12 December 2019 / Accepted: 7 February 2020 / Published online: 25 February 2020 \\ (C) Academic Psychiatry 2020
}

As trainees about to graduate from psychiatry residency, we believe that budding psychiatrists face a key dilemma when considering how to spend our careers: whether to prioritize intervention or innovation. To make the biggest impact, should we focus on applying what is known in psychiatry, delivering care to patients who need it now, expanding access to treatments that work? Or should we devote our resources to advancing our understanding of mental disorders, to discovering novel treatments, to pushing the field in new directions?

Perhaps nowhere is this dilemma more obvious than in the San Francisco Bay Area. Every day, we go to work next to space-age laboratories, where researchers use tools like optogenetics, functional magnetic resonance imaging, and transcranial magnetic stimulation to develop new insights into mental disorders. As part of our training, we receive teaching at the forefront of psychiatry, whether grand rounds about ketamine infusions, lunch talks about the default mode network, or didactics about pharmacogenomics. Yet, beyond the walls of our academic medical center, we too often see people muttering to themselves, wandering the streets in the throes of psychosis. We see tent encampments dotting sidewalks, where people openly inject substances into their necks, and the flashing lights of police cars as officers try to calm someone screaming in the park. We see how far psychiatry has to go.

Psychiatrists-in-training have compelling reasons to focus on intervention. According to the 2018 National Survey on Drug Use and Health, approximately 4.1 million US adults with serious mental illness, out of a total 11.4 million, did not receive any mental health services in the past year [1]. Among an estimated 20.3 million US adults who needed substance use treatment in 2018, just 3.5 million received any in the year prior [1]. More than 47,000 individuals died by suicide in the USA during 2017. Meanwhile, the list of

Nathaniel P. Morris npm@stanford.edu

1 Stanford University School of Medicine, Stanford, CA, USA underutilized, evidence-based treatments in psychiatry is far too long. For instance, research suggests clozapine can decrease psychotic symptoms, suicidality, and mortality among patients with schizophrenia, yet a 2014 study of nearly 80,000 antipsychotic treatment episodes for Medicaid-insured adults with treatment-resistant schizophrenia found just $5.5 \%$ included clozapine initiation over a 4-year period [2]. Electroconvulsive therapy is among the most effective treatments for treatment-resistant mood disorders, yet a study of approximately 1 million privately insured US adults with mood disorders found just $0.3 \%$ had received electroconvulsive therapy in 2014 [3]. Opioid agonist therapy can decrease mortality by half or more for patients with opioid use disorders (OUDs); however, in 2017, 46.4\% of US counties lacked any publicly listed clinician who could offer OUD medications [4].

At the same time, there is an obvious need for innovation in psychiatry. As noted in a 2019 article, "biologic psychiatry has thus far failed to produce a comprehensive theoretical model of any major psychiatric disorder, any tests that can be used in a clinic to diagnose clearly defined major psychiatric disorders, or any guiding principle for somatic treatments to replace the empirical use of medications" [5]. Mental disorders are classified by symptom clusters, yet these labels likely do not reflect the neurobiology underlying these symptoms. We do not understand how the majority of our treatments, including medications and psychotherapies, work, and we have little ability to predict the course of any individual's disease.

No one psychiatrist can take on every role. So how should budding psychiatrists weigh these competing needs for intervention and innovation?

First, psychiatrists-in-training need exposure to both of these domains. During our residency training, we have spent most of our time caring for patients; still, protected time for scholarly work has enabled us to conduct research projects on basic neuroscience, medical education, and psychiatric services. Investing in education that supports both intervention and innovation, including research track residencies, protected scholarly time for non-research residents, and experiences in 
front-line community clinics, can enable psychiatrists to begin thinking about these competing needs during training.

Second, the next generation of psychiatrists can leverage new discoveries to better utilize existing treatments. Telepsychiatry is allowing psychiatrists to provide gold standard treatments, such as buprenorphine for OUD, to patients who live in remote areas and might otherwise not receive care. When properly studied and regulated, mobile applications bring hope for expanding access to evidence-based treatments, including cognitive behavioral therapy and mindfulness exercises. Although still in its infancy, precision psychiatry, using tools like genomics and imaging, offers potential pathways to better match patients with existing treatments.

Third, future psychiatrists can pay greater attention to structural determinants of mental health. Psychiatric training has often overlooked structural factors that affect mental health, including criminalization, racial disparities, unstable housing, and economic marginalization. A psychiatrist can make a difference by helping an individual patient obtain a specific medication; however, by shaping systems of care, including hospital formularies, pharmacy benefit coverage, and electronic prescribing procedures, the same psychiatrist can influence access to this medication for many more patients. Likewise, an innovation-minded psychiatrist might choose to develop her own topics for research, but asking patients what matters to them and their communities can yield new kinds of questions worth studying.

Fourth, psychiatrists need to expand our roles beyond the medical community and to engage more with the public. We cannot work in laboratories and ignore the chronic public underfunding of psychiatric research compared with the burdens of mental disorders. We cannot adequately care for individual patients if we ignore the influence of public stigma on treatment access, insurance reimbursement, and workforce shortages in psychiatry. By working with policymakers, patient organizations, and media, psychiatrists can educate the public about the realities of mental health care and advocate for evidence-based policies. If psychiatrists stay confined to laboratories and clinics, we risk having our research findings gather dust, our nation's mental health policies designed without our input, and our patients shackled by enduring stigma.

The American Psychiatric Association's definition of a psychiatrist largely focuses on direct clinical care [6]. Taking care of patients is a key role of psychiatrists; however, as our residency graduation approaches, we believe this role is just one of many that tomorrow's psychiatrist can embody.

Funding Information Dr. Eshel was financially supported by NIH grant T32 MH019908.

\section{Compliance with Ethical Standards}

This article did not involve research with human participants and, as a result, was not submitted to an institutional review board for approval.

Diclosures On behalf of all authors, the corresponding author states there are no conflicts of interest.

\section{References}

1. Substance Abuse and Mental Health Services Administration. Key Substance Use and Mental Health Indicators in the United States: Results from the 2018 National Survey on drug use and health. Rockville, MD: Center for Behavioral Health Statistics and Quality, Substance abuse and Mental Health Services Administration. https://www.samhsa.gov/data/sites/default/files/ cbhsq-reports/NSDUHNationalFindingsReport2018/ NSDUHNationalFindingsReport2018.pdf. Accessed January 28, 2020.

2. Stroup TS, Gerhard T, Crystal S, Huang C, Olfson M. Geographic and clinical variation in clozapine use in the United States. Psychiatr Serv. 2014;65:186-92.

3. Wilkinson ST, Agbese E, Leslie DL, Rosenheck RA. Identifying recipients of electroconvulsive therapy: data from privately insured Americans. Psychiatr Serv. 2018;69:542-8.

4. Haffajee RL, Lin LA, Bohnert ASB, Goldstick JE. Characteristics of US counties with high opioid overdose mortality and low capacity to deliver medications for opioid use disorder. JAMA Netw Open. 2019;2:e196373.

5. Gardner C, Kleinman A. Medicine and the mind - the consequences of psychiatry's identity crisis. N Engl J Med. 2019;381: 1697-9.

6. American Psychiatric Association. What is psychiatry? https://www. psychiatry.org/patients-families/what-is-psychiatry. Accessed December 10, 2019.

Publisher's Note Springer Nature remains neutral with regard to jurisdictional claims in published maps and institutional affiliations. 